\title{
Competing risks
}

\author{
Jason Fine • Bo Henry Lindqvist
}

Received: 27 December 2013 / Accepted: 25 February 2014 / Published online: 18 March 2014 (C) Springer Science+Business Media New York 2014

The analysis of competing risks data has a long history, traceable to 1766 and the work of Daniel Bernoulli on the impact of smallpox on mortality in 18th century Europe. Today as then, statistical innovation is driven by pressing concerns of society and industry, which must be addressed using complex time to event data. This collection of articles attempts to highlight emerging areas in biomedical and reliability applications where existing methodology is insufficient to address the core issues, and innovative approaches are required. It is indeed an exciting time to be working in this area.

To address mortality issues in the smallpox epidemic, Bernoulli formulated a latent event time model which enabled non-smallpox mortality to be isolated from smallpox mortality, thereby enabling projections which would not have been possible using other formulations. In reliability, this formulation using latent variables continues to play a major role, with systems decomposable into independent components, or maintenance actions being modeled as dependent risks. The papers of Yili Hong and William Q. Meeker, and of Yann Dijoux and Olivier Gaudoin, present methodological innovations tailored to current needs in industrial engineering.

In biomedical applications, the focus has shifted somewhat from the latent variable formulation to the modeling of the observable data, either via the cause specific hazard or cumulative incidence functions. These quantities specify event patterns in the current reality where multiple risks may exist, in contrast to the latent variable formu-

\footnotetext{
J. Fine $(\varangle)$

Department of Biostatistics, University of North Carolina at Chapel Hill, Chapel Hill, NC27599-7400, USA

e-mail: jfine@email.unc.edu

B. H. Lindqvist

Department of Mathematical Sciences, Norwegian University of Science and Technology, 7491Trondheim, Norway

e-mail: bo@math.ntnu.no
} 
lation, where the latent event time for a particular risk is interpreted in a hypothetical reality where all other risks have been eliminated. The cause specific hazard captures instantaneous risks which may be useful biologically, while the cumulative incidence functions define absolute risks which may be of importance to patients, clinicians and policy makers. Ravi Varadhan, Qian-Li Xue and Karen Bandeen-Roche provide a critical assessment of both formulations in aging research, where competing risks are critically important because of censoring by mortality, and review different methodological strategies. These analytic choices are critically important in understanding population dynamics and planning for future health care needs.

A primary theme of this volume is the extension of methods for independently censored data to the competing risks setting. The potentially dependent censoring arising from the competing risks, in addition to the usual independent censoring, may either complicate or invalidate the use of methods developed for independently censored data. A number of papers consider design issues where the cause-specific hazard and cumulative incidence are of primary interest. The assessment of association and its impact on predictions in family based studies involving disease endpoints censored by mortality is discussed in the paper by Thomas H. Scheike, Klaus K. Holst and Jacob B. Hjelmborg, and also in the paper by Malka Gorfine, Li Hsu, David M. Zucker and Giovanni Parmigiani, extending previous work on data without competing risks. Similarly, complex sampling designs which have been addressed for independently censored data are here considered for competing risks, with Stephanie A. Kovalchik and Ruth M. Pfeiffer addressing survey designs; Jean-Yves Dauxois, Agathe Guilloux and Syed N.U.A. Kirmani addressing length-biased sampling; and Nadine Binder, Thomas A. Gerds and Per Kragh Andersen, as well as Liang Li, Bo $\mathrm{Hu}$ and Michael W. Kattan, addressing covariate dependent censoring, as may occur in nonrandomized cohort studies or adaptive clinical studies.

Finally, the contributions of Arthur Allignol, Jan Beyersmann, Thomas A. Gerds and Aurélien Latouche, and of Laura L. Taylor and Edsel A. Pena, study nonparametric estimation with competing risks embedded in more complicated data structures, respectively the non-Markov illness death model and recurrent competing risks data. As with the aforementioned papers, these works respond directly to the complexity of data arising in both biomedical and reliability applications.

We trust that you will find these papers stimulating, representing the state of the art in both the theory and application of competing risks methodology. Looking back almost 250 years, our hope is that in 250 years, this volume may permit one to trace the arc of developments in competing risks, recognizing the importance of applications in providing direction to these developments, and placing today's work in historical context.

We would finally like to thank the authors for their excellent contributions to this special issue. In fact, because of the overwhelming positive response from the invited participants, we were, due to page limits, not able to include all the papers in one issue. The papers by Varadhan, Xue and Bandeen-Roche on semicompeting risks in aging research; by Allignol, Beyersmann, Gerds and Latouche on the non-Markov illnessdeath model; and by Taylor and Pena on recurrent competing risks, will therefore appear in the next issue. 